\title{
Caracterización de los casos de accidente ofidico atendidos por el Centro de Información y Estudio de Medicamentos y Tóxicos (CIEMTO) de Medellin, Colombia durante 2016
}

\author{
Characterization of snakebite cases attended by the \\ drug and poison research and information center \\ (CIEMTO) in Medellin, Colombia during 2016
}

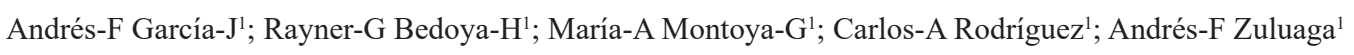

Forma de citar: García A, Bedoya R, Montoya M, Rodríguez C, Zuluaga A. Caracterización de los casos de accidente ofídico atendidos por el Centro de Información y Estudio de Medicamentos y Tóxicos (CIEMTO) de Medellín, Colombia durante 2016. Rev Univ Ind Santander Salud. 2017; 49(3): 450-457. doi: http://dx.doi.org/10.18273/revsal.v49n3-2017003 (c) (1)

\section{Resumen}

Introducción: Las mordeduras de serpiente continúan siendo un problema de salud pública, especialmente en países tropicales como Colombia. Objetivo: Caracterizar los casos de accidente ofídico atendidos en un nuevo centro de asesoría toxicológica de Medellín, Colombia. Metodología: Se realizó un estudio descriptivo, retrospectivo, revisando la base de datos donde se registra la información relacionada con la asesoría brindada por dicho centro desde el 1 de enero hasta el 31 de diciembre de 2016. Resultados: Se registraron 117 casos de accidente ofídico, de los cuales 93 (79\%) eran hombres y 24 (21\%) mujeres, con una mediana de edad de 32 años (rango: 2 a 82 años). El seguimiento de los casos pudo lograse en 55 de los 117 accidentes (47\%), y se describieron complicaciones en 18 de los 55 (33\%) pacientes. La complicación descrita con mayor frecuencia fue brote maculopapular pruriginoso asociado con la administración del suero, sin que se identificaran diferencias entre las distintas marcas de suero antiofídico utilizado. Se documentó la muerte de un paciente (0,85\%). El género Bothrops produjo la mayoría de los accidentes. Discusión: Los datos obtenidos coinciden con la bibliografía publicada.

Palabras clave: Mordeduras de serpientes; Animales venenosos; Colombia; Enfermedad del suero; Toxicología.

\begin{abstract}
Introduction: Snakebites continue to be a public health problem, especially in tropical countries like Colombia. Objetive: To characterize the snakebite cases attended by a new poison center in Medellin, Colombia. Methodology: A descriptive, retrospective study was carried out, reviewing the information of the Center's database from January

1. Universidad de Antioquia, Medellín, Colombia

Correspondencia: Andrés F. Zuluaga. Dirección: Calle 67 No 53-108 Medellin. Antioquia. Colombia. Correo electrónico: andres.zuluaga@ udea.edu.co. Teléfono: +5742196046.
\end{abstract}


$1^{\text {st }}$ to December $31^{\text {st }}, 2016$. Results: There were 117 cases of ophidian accidents, affecting 93 men (79\%) and 24 women (21\%), with a median age of 32 years (range: 2 to 82 years). The follow-up of the cases could be done in 55 of the 117 accidents (47\%), and complications were described in 18 of those 55 (33\%) patients. The most commonly reported complication was a pruritic maculopapular rash that was associated to serum administration, without differences between the several brands of anti-ophidian serum used. The death of one patient $(0.85 \%)$ was documented. The genus Bothrops caused most of the accidents. Discussion: Our results agree with previously published data.

Keywords: Snakebites; Animals; Poisonous; Colombia; Serum sickness; Toxicology.

\section{Introducción}

Los accidentes ofídicos continúan siendo un problema de salud pública, especialmente en países tropicales ${ }^{1,2}$. La Organización Mundial de la Salud estima que anualmente 5 millones de personas sufren mordeduras por serpientes, de ellas más de 100.000 mueren y hasta 400.000 sufren amputaciones ${ }^{3}$. En Colombia, el Instituto Nacional de Salud (INS) reportó que durante el 2016 se presentaron 4636 casos de accidente ofídico (con una tendencia creciente en comparación con años anteriores); en los cuales se informaron 31 defunciones, 16 no fueron hospitalizadas y en los 15 decesos adicionales no se suministró suero antiofídico ${ }^{4}$.

En pleno siglo XXI, las comunidades rurales de África, Asia y Latinoamérica continúan siendo las más afectadas, y de manera particular las personas en edad productiva ${ }^{5}$. El pronóstico de estos accidentes está directamente relacionado, con la práctica de medidas artesanales prehospitalarias (succión de la herida o aplicación emplastos), la administración oportuna y correcta del suero antiofídico específico y el uso de tratamientos no indicados como antibióticos ${ }^{6}$. Sin embargo, es escasa la formación médica sobre toxicología en países como Colombia, lo cual podría favorecer la aparición de desenlaces indeseados en los pacientes.

Los centros de información de medicamentos y tóxicos son unidades especializadas que brindan asesoría sobre la prevención, el diagnóstico y el manejo médico adecuado de las intoxicaciones ${ }^{8}$. En Colombia existen seis centros de información toxicológica distribuidos en el territorio nacional. En Medellín desde hace dos años opera el Centro de Información y Estudio de Medicamentos y Tóxicos (CIEMTO) de la Universidad de Antioquia ${ }^{7}$.

Durante 2016, CIEMTO brindó asesoría médica especializada mediante tecnologías de la información y la comunicación en 117 casos de pacientes que sufrieron mordeduras por serpientes. El objetivo general de este trabajo es caracterizar dichos casos de accidente ofídico atendidos en CIEMTO durante 2016 y evaluar su desenlace clínico, con el fin de analizar de qué el impacto de las intervenciones sugeridas en la evolución de los pacientes.

\section{Métodos}

Se realizó un estudio descriptivo, retrospectivo, anidado en la base de datos donde se registra la información relacionada con la asesoría brindada en CIEMTO. Se emplearon inicialmente todos los casos atendidos a través de llamadas telefónicas realizadas entre el 1 de enero y el 31 de diciembre de 2016. Las llamadas telefónicas fueron atendidas y registradas por médicos con un mínimo de dos años de formación en toxicología clínica. De la totalidad de casos asesorados, se seleccionaron los que incluyeron algún tipo de diagnóstico relacionado con accidentes por animales ponzoñosos, y posteriormente se incluyeron para este estudio los casos de accidente por mordedura de serpiente.

Previamente se obtuvo el consentimiento informado para el registro de datos utilizados durante la atención o la asesoría, posteriormente almacenados en una aplicación web. Las variables a tener en cuenta fueron: edad, género, departamento y ciudad de donde se originó la llamada, tipo de serpiente, localización anatómica de la lesión, clasificación del accidente ofídico, medidas prehospitalarias, uso y tipo de suero antiofídico. El seguimiento se hizo a través de llamada telefónica dirigida al médico tratante del sitio remisor, y se indago por desenlaces como mortalidad, complicaciones infecciosas o no infecciosas y secuelas; estos datos se incluyeron en la base de datos final usada para el análisis, elaborada en Excel (Microsoft, USA). Salvo se exprese lo contrario, los resultados son presentados como medianas con rangos mínimo a máximo y frecuencias (porcentajes). 


\section{Resultados}

Durante 2016, se solicitó asesoría médica especializada en farmacología y toxicología en CIEMTO para 1440 casos, $225(16 \%)$ de los cuales fueron atribuidos a accidentes por animales ponzoñosos. La Figura 1 describe el tipo de accidente atendido, de acuerdo con el animal involucrado.

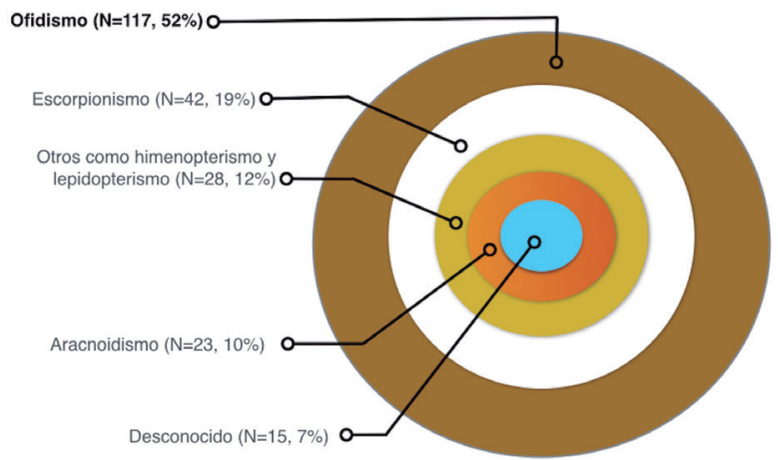

Figura 1. Tipos de accidente por animales ponzoñosos.

Los animales involucrados con mayor frecuencia fueron, en su orden serpientes, escorpiones, abejas, mariposas y arañas. En los casos agrupados como desconocidos, ni los pacientes ni el profesional de la salud pudieron identificar o reportar el animal involucrado, pero las características clínicas y epidemiológicas eran sugestivas de un envenenamiento por animal ponzoñoso.

En la Figura 2 se distribuyen los 117 casos de accidente por mordedura de serpiente incluidos en el estudio. En total, se reportaron $93(79 \%)$ hombres y $24(21 \%)$ mujeres afectados, con una mediana de edad de 32 años (rango: 2 a 82 años). El tiempo transcurrido entre el accidente ofídico y el primer reporte o solicitud de asesoría en CIEMTO tuvo una mediana de siete horas, con rango mínimo de media hora y máximo de 168 horas.

Se pudo realizar seguimiento al $47 \%$ de los 117 casos atendidos. En total se reportaron 21 pacientes con complicaciones, la mayoría (10 casos) por brotes tras la aplicación del suero antiofídico. Solo se presentó una fatalidad.

Solo se reportó un caso de accidente ofídico en una paciente con embarazo de siete semanas. En ese caso no se pudo identificar el género de la serpiente, se le realizó prueba del todo o nada la cual coaguló y no requirió manejo con antiveneno, ni presentó complicaciones. La identificación del género de las serpientes agresoras pudo ser efectuada en 94 de los 117 casos (80\%), gracias a la información suministrada por los pacientes (verbalmente o por fotografías), o por el equipo de salud que solicitaba la asesoría a CIEMTO. Entre los casos en que se describió el tipo de animal involucrado, el género de serpiente que más se reportó fue Bothrops spp. ( $\mathrm{N}=83,88 \%$ ), seguido por Micrurusspp. $(\mathrm{N}=6,6 \%)$, el resto de casos correspondieron a especies clasificadas como no venenosas, tipo colúbridos $(\mathrm{N}=5,6 \%)$.

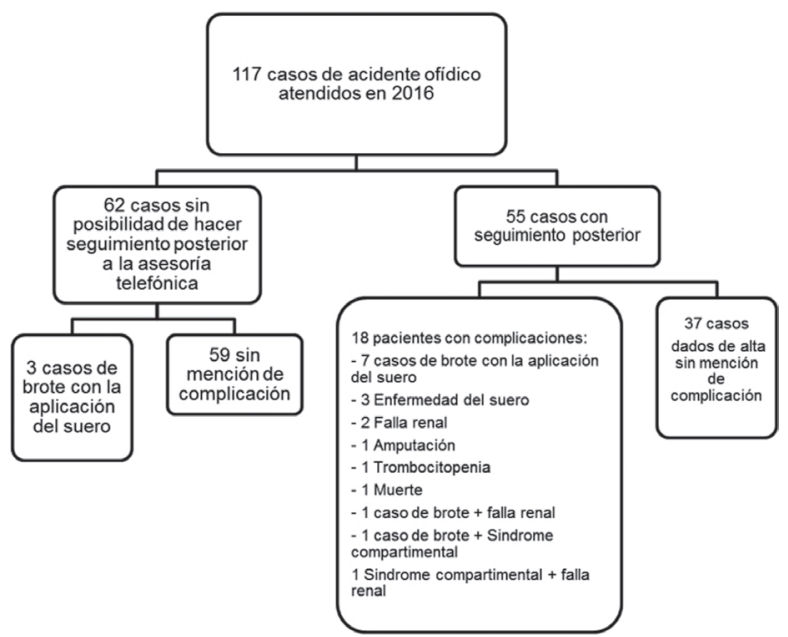

Figura 2. Caracterización de los accidentes ofídicos atendidos en la línea CIEMTO en 2016.

Entre los pacientes que tuvieron seguimiento telefónico $(\mathrm{N}=55,47 \%)$, se describieron complicaciones en 1 de cada 3 casos (Figura 2). El brote maculopapular pruriginoso (rash) fue la complicación más común, y se relacionó con la administración del suero, sin que se identificaran diferencias entre las distintas marcas de suero antiofídico utilizado. Veinte de los 55 (36\%) casos, tuvieron que ser referidos a un nivel de mayor complejidad hospitalaria. Se documentó la muerte de un paciente, tres casos describieron insuficiencia renal, dos pacientes más presentaron síndrome compartimental en el miembro superior, uno requirió amputación de un dedo de la mano y en otro paciente se documentó trombocitopenia (50.000 células $/ \mu \mathrm{L}$ ). No se presentó ningún caso de infección en el seguimiento realizado a los pacientes ni de hemorragia del sistema nervioso central.

La Figura 3 muestra la distribución de las llamadas por región, dentro del Departamento de Antioquia, Colombia; donde se originaron 94 de las $117(80 \%)$ solicitudes de asesoría a nivel nacional. La región donde más casos se reportaron a CIEMTO fue el Suroeste con 22 casos, desde los municipios de Ciudad Bolívar, Salgar y Urrao. La región desde la que se recibieron menos reportes fue la del Bajo Cauca, con sólo tres casos en el 2016. En general,la mayoría de llamadas para asesoría especializada provinieron de instituciones de salud pertenecientes al sector público ( $\mathrm{N}=108,92 \%)$. 


\begin{tabular}{|l|c|l|l|}
\hline Región de Antioquia & \# de llamadas & \multicolumn{1}{|c|}{ Municipio con más casos (N) } \\
\hline Suroeste & $\mathbf{2 2}$ & Ciudad Bolivar (6) \\
\hline Nordeste & 15 & Anori (7) \\
\hline Norte & 15 & Ituango (7) \\
\hline Oriente & 15 & San Luis (7) \\
\hline Occidente & 8 & Varios \\
\hline Valle de Aburrá & 8 & Medellín y área metropolitana (8) \\
\hline Magdalena medio & 4 & Puerto Triunfo (2) \\
\hline Urabá & 4 & Apartadó (2) \\
\hline Bajo Cauca & 3 & Varios \\
\hline
\end{tabular}

Figura 3. Distribución de las llamadas por región dentro del Departamento de Antioquia, Colombia.

En Antioquia se atendieron 94 casos. Excepto por Medellín, los hospitales donde fueron atendidos los pacientes en general tenían niveles de complejidad medio o bajo (segundo o primer nivel de atención). Los restantes 23 casos asesorados se originaron en otros Departamentos de Colombia.
La Figura 4 muestra la distribución por gravedad del accidente ofídico. El sitio anatómico más afectado fueron los miembros superiores ( $\mathrm{N}=61,52 \%)$, seguido por los miembros inferiores ( $\mathrm{N}=48,41 \%)$, la cabeza $(\mathrm{N}=2,2 \%)$ y en seis casos $(5 \%)$ no se registró el sitio de la mordedura.

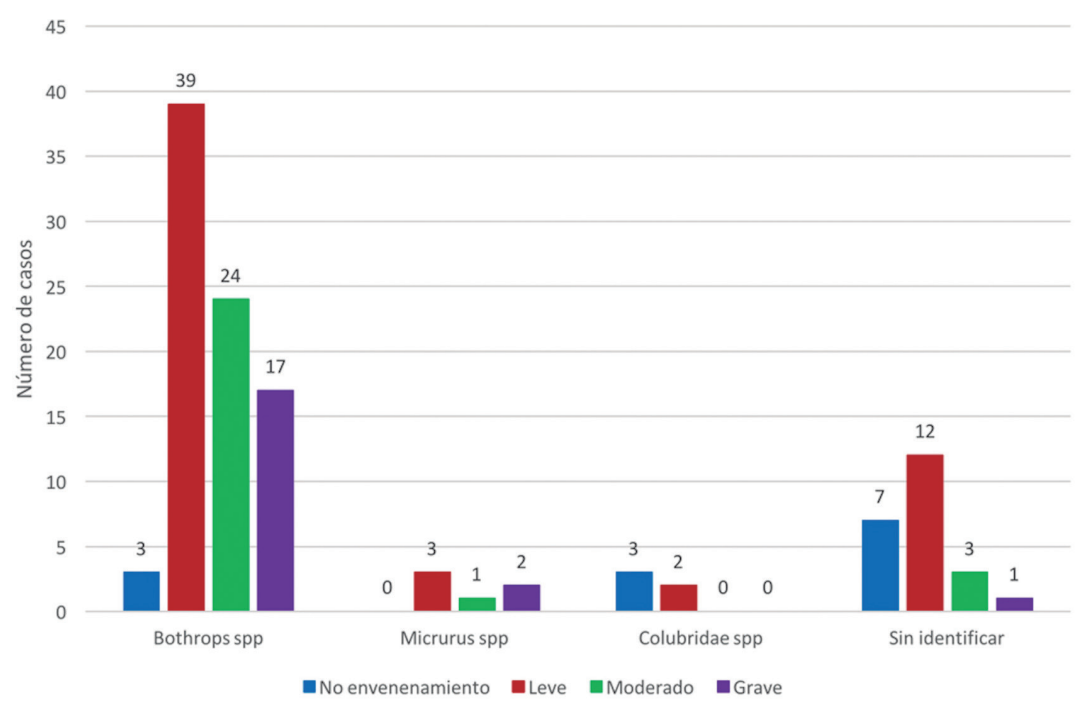

Figura 4. Distribución por tipo de serpiente y gravedad de los accidentes ofídicos.

La mayoría de accidentes con serpiente identificada fueron por el género Bothrops, seguido por Micrurus y la familia Colubridae. La serpiente no se pudo identificar en 23 casos. En todos los grupos predominaron los accidentes leves a moderados.

En 22 de los 117 casos (19\%), se describió que se practicaron antes de la atención médica, medidas artesanales como aplicación de torniquetes $(\mathrm{N}=10,45 \%)$, administración de pócimas o bebidas naturales $(\mathrm{N}=5$, $22 \%)$, emplastos $(\mathrm{N}=4,18 \%)$ y en los casos restantes se hicieron incisiones en el sitio de la mordedura $(\mathrm{N}=3$,
$14 \%)$. Por otra parte, en 72 casos (62\%) se administró algún tipo de suero antiveneno. La Tabla 1 caracteriza el antiveneno utilizado en estos casos, especificando la marca utilizada. En total se usaron 368ampollas, con una mediana de cuatro ampollas por paciente, y un rango entre 1 y 24 ampollas aplicadas. De acuerdo con la gravedad del accidente ofídico, la mediana de las ampollas aplicadas fue: 4 ampollas en los casos leves y moderados y 6 ampollas en los casos graves.

En la Tabla 2 se presenta un resumen de los hallazgos más relevantes del estudio. 
Caracterización de los casos de accidente ofídico atendidos por el Centro de Información y Estudio de Medicamentos y Tóxicos (CIEMTO) de Medellín, Colombia durante 2016

Tabla 1. Caracterización de los antivenenos empleados.

\begin{tabular}{|c|c|c|c|c|}
\hline $\begin{array}{l}\text { Género de } \\
\text { serpiente }\end{array}$ & $\begin{array}{c}\text { Uso de } \\
\text { antiveneno }\end{array}$ & $\begin{array}{l}\text { Marca del antiveneno o } \\
\text { proveedor }\end{array}$ & $\begin{array}{c}\text { Tipo de antiveneno (géneros usados para } \\
\text { inmunizar los caballos de donde proviene el suero) }\end{array}$ & $\begin{array}{l}\text { Número de } \\
\text { llamadas }\end{array}$ \\
\hline \multirow{7}{*}{$\begin{array}{l}\text { Bothrops spp } \\
(\mathrm{N}=83)\end{array}$} & \multirow{6}{*}{ Sí } & $\begin{array}{l}\text { Antivipmyn TRITM } \\
{\text { (faboterápico })^{\mathrm{a}}}\end{array}$ & Fragmentos F(ab')2 (Bothrops, Crotalus y Lachesis) & 20 \\
\hline & & $\begin{array}{l}\text { Instituto Nacional de } \\
\text { Salud (INS) }{ }^{\mathrm{b}}\end{array}$ & $\begin{array}{l}\text { Inmunoglobulina G específica, purificada, (Bothrops y } \\
\text { Crotalus) }\end{array}$ & 15 \\
\hline & & Probiol $^{\mathrm{c}}$ & Inmunoglobulina G (Bothrops, Lachesis y Crotalus) & 5 \\
\hline & & $\begin{array}{l}\text { Instituto Clodomiro } \\
\text { Picado }^{\mathrm{d}}\end{array}$ & Inmunoglobulina G (Bothrops, Lachesis y Crotalus) & 1 \\
\hline & & INS y Antivipmyn TRITM & Ver cada producto individual & 1 \\
\hline & & Sin datos & No aplica & 23 \\
\hline & No & No aplica & No aplica & 18 \\
\hline \multirow{2}{*}{$\begin{array}{l}\text { Micrurus spp } \\
(\mathrm{N}=6)\end{array}$} & Sí & Sin datos & No aplica & 3 \\
\hline & No & No aplica & No aplica & 3 \\
\hline \multirow{3}{*}{$\begin{array}{l}\text { Colubridae } \\
(\mathrm{N}=5)\end{array}$} & Sí & Sin datos & No aplica & 1 \\
\hline & No & No aplica & No aplica & 4 \\
\hline & & Antivipmyn TRI ${ }^{\mathrm{TM}}$ & Ver descripción previa & 2 \\
\hline \multirow{4}{*}{$\begin{array}{l}\text { No identificada } \\
(\mathrm{N}=23)\end{array}$} & \multirow[t]{3}{*}{ Sí } & $\begin{array}{l}\text { Instituto Clodomiro } \\
\text { Picado }\end{array}$ & Ver descripción previa & 1 \\
\hline & & INS & Ver descripción previa & 1 \\
\hline & & Sin datos & Ver descripción previa & 1 \\
\hline & No & No aplica & No aplica & 18 \\
\hline
\end{tabular}

Fuentes (accedidas el 30/06/2017):

$\mathrm{a}=\mathrm{http}: / /$ www.comercializadorafarma.com/image.ashx?i=216763.pdf\&fn=

b=http://www.ins.gov.co/Documents/INFORMACION\%20SUERO\%20ANTIOFIDICO\%20POLIVALENTE.pdf

$\mathrm{c}=\mathrm{http}: / / \mathrm{www} \cdot$ probiol.com/images/pdf/probiolsueropolivalente.pdf

$\mathrm{d}=$ http://icp.ucr.ac.cr/recursos/docs/sueros/INSERTO_POLQ_POLF.pdf

Tabla 2. Resumen de once hallazgos relevantes del estudio.

\begin{tabular}{lc}
\hline \multicolumn{1}{c}{ CARACTERÍSTICA } & RESULTADO \\
\hline Número de accidentes ofídicos asesorados durante 2016 en CIEMTO (\%) & $117(100 \%)$ \\
Mediana de edad (años) de la población afectada (rango mínimo a máximo) & $32(2$ a 82$)$ \\
Número de hombres afectados (\%) & $93(79 \%)$ \\
Mediana del tiempo (horas) transcurrido entre el accidente y solicitud de asesoría en CIEMTO & 7 (0.5-168) \\
(rango mínimo a máximo) & 1 \\
Número de mujeres embarazadas afectadas & 94 (80) \\
Número de casos en los cuales se identificó la serpiente involucrada (\%) & 83 (88) \\
Número de casos que describieron accidente bothrópico (\%) & 55 (47) \\
Número de casos con seguimiento telefónico posterior (\%) & 18 (33) \\
Número de casos que manifestaron complicaciones durante el seguimiento (\%) & 9 (50) \\
Número de casos complicados por brote maculopapular pruriginoso (\%) & 1 (2) \\
Número de pacientes que fallecieron durante el seguimiento (\%) & 108 (92) \\
Número de llamadas a CIEMTO que provinieron del sector público (\%) & 21 (18) \\
Número de pacientes con accidente ofídico grave (\%) & $22(19)$ \\
Número de casos en los que se reportó uso de medidas artesanales (\%) & (62) \\
Número de pacientes en los que se reportó la administración de antiveneno (\%) & 4 (1 a 24) \\
\hline
\end{tabular}




\section{Discusión}

Este trabajo es el primer análisis epidemiológico de los casos de accidente ofídico atendidos en la línea CIEMTO, apoyándose en herramientas propias de la telemedicina. Según el Instituto Nacional de Salud de Colombia (INS), la incidencia de accidenteofídico en el país durante el año 2016 fue de 10 casos por cada 100 mil habitantes. Antioquia fue el Departamento de Colombia donde mayor número de casos se reportaron al INS en 2016, con un total de 721 casos. Por tanto, CIEMTO asesoró la atención médica de al menos uno de cada ocho accidentes (94 de 721 casos, 13\%) que se presentaron en ese Departamento durante 2016.

El mayor reporte de casos en el departamento de Antioquia puede explicarse por una mejor notificación de los casos debido a la mejor gestión del sistema de salud y también por la diversa fauna de las serpientes que allí se encuentran. El INS ha venido realizando capacitaciones a nivel nacional, lo cual se evidencia en el aumento de la notificación en los últimos años.

El accidente por serpientes del género Bothrops (88\%) fue el más reportado en la línea CIEMTO, semejante a reportes de otros países de la región como $\mathrm{Brasil}^{8} \mathrm{y}$ Venezuela ${ }^{9}$ y lo reportado por el INS. Este predominio de los ataques de Bothrops se ha atribuido a la alta diversidad de especies de este género de serpientes, su agresividad, al hecho de que están ampliamente distribuidos en Colombia y pueden encontrarse en la mayoría de los ecosistemas ${ }^{10}$.

Aún existe un número considerable de casos $(20 \%)$ donde no fue posible identificar la serpiente, lo que puede indicar falta de conocimientos técnicos del personal de salud y de la población en general para determinar las características específicas de cada género de serpiente e iniciar el antídoto correcto. En estos casos el tratamiento se realizó con base en los hallazgos clínicos referidos por el personal médico que llamó a la línea y las indicaciones de la línea CIEMTO.

El antiveneno fue administrado al $62 \%$ de los casos, es decir que uno de cada tres pacientes no recibió suero antiofídico. En 2016 se reportó al SIVIGILA un $78 \%$ de uso de antiveneno, lo cual es bastante inferior comparado con reportes de Brasil donde la frecuencia es del $94 \%{ }^{7}$, una posible explicación a este fenómeno es que países como Brasil, Costa Rica, México y Argentina son autosuficientes en el abastecimiento de antivenenos ${ }^{11}$. Al contrario, Colombia, Venezuela, Perú y Bolivia, tienen centros productores de antiveneno que no alcanzan a satisfacer la demanda nacional de estos productos ${ }^{11}$. La meta establecida por el INS es que el $100 \%$ de los accidentes ofídicos reciban suero antiveneno. Es necesario entonces implementar medidas que permitan lograr esta meta. En este estudio, como en otros encontrados en la literatura ${ }^{8,12-15}$, la mayoría de las víctimas fueron hombres, quienes realizan la mayor parte de las actividades rurales agrícolas.

Es importante además mencionar el número de casos en los cuales se realizaron prácticas no médicas, siendo uno de los principales objetivos de brindar asesoría toxicológica especializada ir erradicando estas conductas inadecuadas que generan complicaciones y que disminuyen el pronóstico de los pacientes ${ }^{16}$.

Los centros hospitalarios desde donde se realizaron la mayor parte de las llamadas corresponden a centros de primer nivel de atención, los cuales, por lo general no cuentan con todos los recursos necesarios ni con el personal capacitado para el manejo del accidente ofídico, lo que lleva a realizar remisiones de pacientes a centros de mayor complejidad, retrasando el inicio oportuno de la terapia. En la medida que CIEMTO puede asesorar estos casos se disminuyen las remisiones no indicadas, facilitando el inicio rápido del tratamiento.

Durante las llamadas de seguimiento se pudo establecer la muerte de un paciente, lo que corresponde al 1,8\% de los casos atendidos con seguimiento, y que junto al $0,6 \%$ reportado a nivel nacional en el año $2016^{5}$ constata que este evento tiene en general una baja mortalidad. No obstante, la conocida variabilidad geográfica y las diferencias intraespecíficas de los venenos de las serpientes que se han descrito por técnicas electroforéticas, también pueden explicar la diferencia en mortalidad respecto al promedio naciona ${ }^{17}$.

La formalización de una estrategia para el manejo de la mordedura de serpiente y la concientización del personal de salud y de la población en general, deben llevarse a cabo para mejorar el sistema de notificación de casos y mejorar los desenlaces. Uno de los objetivos de CIEMTO es lograr un mejor conocimiento de dicho evento, no solo en el personal médico, sino también en el público, por medio de las tecnologías de la información y la comunicación (TIC). Es esperable que con la progresiva difusión de la existencia de este centro de información toxicológica se logre este propósito.

Entre las cualidades que deben tener los centros de información toxicológica se encuentra la capacidad de realizar seguimiento a los casos atendidos, pues esto permite evaluar el desenlace de los pacientes, la pertinencia de las recomendaciones brindadas e incluso 
evidenciar errores terapéuticos ${ }^{18}$. En este estudio se realizaron llamadas de seguimiento a todos los casos, logrando establecer el desenlace de los pacientes solo en el $47 \%$ de los casos. Entre las causas de no haber podido completar el seguimiento en el $100 \%$ de los casos están: no lograr comunicación nuevamente con el médico que solicitó la asesoría, cambios laborales en la institución de salud desde la que hizo la llamada, y restricciones administrativas para entregar la información por parte de las instituciones de salud. Esta es una de las principales limitaciones porque nos impide hacer comparaciones con otros centros de información que tienen tasas de seguimiento más altas ${ }^{19}$. Para mejorar esta situación, el seguimiento obligatorio y oportuno de cada uno de los casos recibidos en la línea CIEMTO se implementará a partir del segundo trimestre de 2017. Otra de las debilidades del presente estudio es su carácter retrospectivo, además, los datos analizados corresponden a la base de datos de CIEMTO y no representan la estadística total registrada sobre ofidismo en Antioquia o en Colombia, por lo cual debe tenerse precaución al extrapolar sus resultados a nivel departamental o nacional.

En conclusión, las mordeduras de serpiente continúan encabezando los accidentes por animales ponzoñosos y generando un impacto en la población en edad productiva, como se confirma en este estudio cuyos resultados concuerdan con los datos a nivel nacional. CIEMTO ha realizado ingentes esfuerzos para mejorar la atención de los casos de accidente ofídico en la población colombiana utilizando tecnologías de la información y la comunicación. En la actualidad, las acciones deben centrarse en ampliar el reconocimiento de la línea toxicológica a nivel nacional, la optimización de la base de datos y el reforzamiento del seguimiento de todos los casos, con miras a brindar una mejor asesoría y contribuir a reducir la morbilidad y mortalidad por accidente ofídico.

\section{Agradecimientos}

Los autores desean agradecer a todo el personal de CIEMTO, por su empeño en buscar soluciones en su campo que permitan mejorar o salvar vidas.

\section{Referencias}

1. Zambrano-Ospina ÁM. Accidente ofídico como evento de interés en salud pública en Colombia: aportes al diseño de estrategias de gestión(Tesis de maestría). Universidad Nacional de Colombia. Bogotá, 2012.
2. Gutiérrez JM, Williams D, Fan HW, Warrell DA. Snakebite envenoming from a global perspective: Towards an integrated approach. Toxicon. 2010; 56(7): 1223-1235. DOI: 10.1016/j. toxicon.2009.11.020.

3. Arnold BYC. The snakebite fight. Nature. 2016; 537: 6-8.

4. Instituto Nacional de Salud, Colombia. Informe del evento accidente ofídico hasta el periodo epidemiológico XIII, Instituto Nacional de Salud, Colombia 2016.

5. Harrison RA, Gutiérrez JM. Priority actions and progress to substantially and sustainably reduce the mortality, morbidity and socioeconomic burden of tropical snakebite. Toxins (Basel). 2016; 8(12): 351.

6. Cuesta J, Peña LM, Zuluaga AF. ¿Es necesaria la profilaxis antibiótica en la ofidiotoxicosis? Infectio. 2008; 12(1): 54-63.

7. Zuluaga AF, Rodriguez CA, Lastra-Bello S, PeñaAcevedo LM, Montoya-Giraldo MA. CIEMTO: the new drug and poison research and information center in Medellín, Colombia. Clin Toxicol (Phila). 2017; 13: 1-2. DOI: 10.1080/15563650.2017.1312003.

8. Leite RDS, Tamarys I, Targino G, Arianne Y, Ferreira C, Barros RM, et al. Epidemiology of snakebite accidents in the municipalities of the state of Paraíba, Brazil. Cien Saude Colet. 2013; 18(5): 1463-1471.

9. De Sousa L, Bastouri-Carrasco J, Matos M, Borges A, Bónoli S, Vásquez-Suárez A, et al. Epidemiología del ofidismo en Venezuela (1996-2004). Investig Clin. 2013; 54(2): 123-137.

10. Gutierrez JM. Envenenamientos por mordeduras de serpientes en América Latina y el Caribe: Una visión integral de carácter regional. Bol Mal Salud Amb. 2011; LI(1): 1-16.

11. Gutiérrez JM. Understanding and confronting snakebite envenoming: The harvest of cooperation. Toxicon. 2016; 109: 51-62.

12. Yañez-Arenas C, Yañez-Arenas A, Martínez-Ortiz D. Panorama epidemiológico de las mordeduras por serpiente venenosa en el estado de Yucatán, México (2003-2012). Gac Med Mex. 2016; 152(4): 568574.

13. Vera, A.; Páez, M.; Gamarra de Cáceres, G. Caracterización Epidemiológica de los accidentes ofídicos, Paraguay 2004. Mem Inst Investig Cienc. Salud. 2006. 4(1): 20-24.

14. Rodríguez-Vargas AL. Comportamiento general de los accidentes provocados por animales venenosos en Colombia, 2006-2010. Rev Salud Pública. 2012. 14(6): 101-110.

15. Márquez Gómez MA, Gómez Díaz GM. Accidente 
ofídico en el departamento de Sucre, Colombia. NOVA. 2015; 13(24): 39-46.

16. Cubides S. Actitudes, creencias y prácticas desarrolladas en comunidades rurales del Urabá antioqueño con relación a las serpientes y su mordedura Municipio de Necoclí, Población Campesina y Comunidad Indígena Senú (Tesis de maestría). Universidad de Antioquia. Medellín, 2016.

17. Gutiérrez, J.M. Comprendiendo los venenos de serpientes: 50 años de investigaciones en América Latina. Rev Biol Trop. 2002; 50: 377-394.

18. Litovitz TL, Elshami JE. Poison center operations: The necessity of follow-up. Ann Emerg Med. 1982; 11(7): 348-352.

19. Mowry JB, Spyker DA, Brooks DE, Zimmerman A, L. SJ. 2015 Annual Report of the American Association of Poison Control Centers' National Poison Data System (NPDS): 33rd Annual Report. ClinToxicol (Phila). 2016; 54(10): 924-1109. 